\title{
Novel scenario for production of heavy flavored mesons in heavy ion collisions
}

\author{
B.Z. Kopeliovich ${ }^{1 a}$, J. Nemchik ${ }^{2,3}$, I.K. Potashnikova ${ }^{1}$, and Ivan Schmidt ${ }^{1}$ \\ ${ }^{1}$ Departamento de Física, Universidad Técnica Federico Santa María; and \\ Centro Científico-Tecnológico de Valparaíso; Casilla 110-V, Valparaíso, Chile \\ ${ }^{2}$ Czech Technical University in Prague, FNSPE, Břehová 7, 11519 Prague, Czech Republic \\ ${ }^{3}$ Institute of Experimental Physics SAS, Watsonova 47, 04001 Kosice, Slovakia
}

\begin{abstract}
The observed strong suppression of heavy flavored hadrons produced with high $p_{T}$, is caused by final state interactions with the created dense medium. Vacuum radiation of high- $p_{T}$ heavy quarks ceases at a short time scale, as is confirmed by pQCD calculations and by LEP measurements of the fragmentation functions of heavy quarks. Production of a heavy flavored hadrons in a dense medium is considerably delayed due to prompt breakup of the hadrons by the medium. This causes a strong suppression of the heavy quark yield because of the specific shape of the fragmentation function. The parameter-free description is in a good accord with available data.
\end{abstract}

\section{Introduction}

In the popular scenario, explaining jet quenching, observed in heavy ion collisions, by induced energy loss in the hot medium created in the nuclear collision, a much weaker suppression, compared with light hadrons, was anticipated [1] for heavy flavors, caused by the dead-cone effect.

Later, however, measurements revealed similar magnitudes of suppression for heavy and light hadrons. Here we propose an alternative scenario for production of heavy flavored hadrons from a hot medium. The novel mechanism well explains data in a parameter-free way.

\section{Hard parton collision}

High-pt parton-parton scattering leads to formation of 4 cones of gluon radiation: (i) the color field of the colliding partons is shaken off in forward-backward directions; (ii) the scattered partons carrying no field up to transverse frequences $k<p_{T}$, are regenerating the lost components of their field, radiating gluons and forming two high- $p_{T}$ jets. This process is illustrated in figure 1 .

The coherence length/time of gluon radiation by a quark of mass $m_{q}$ and energy $E_{q}$ has the form,

$$
l_{c}=\frac{2 E_{q} x(1-x)}{k_{T}^{2}+x^{2} m_{q}^{2}},
$$

ae-mail: boris.kopeliovich@usm.cl 


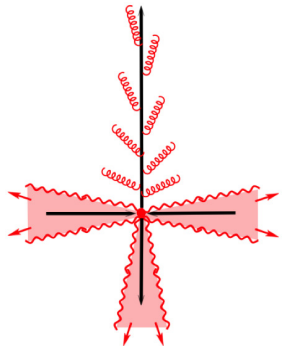

Figure 1. High- $p_{T}$ parton scattering. The two forwardbackward jets are formed by the shaken-off gluon fields. The high- $p_{T}$ partons regenerate the lost field, radiating gluons.

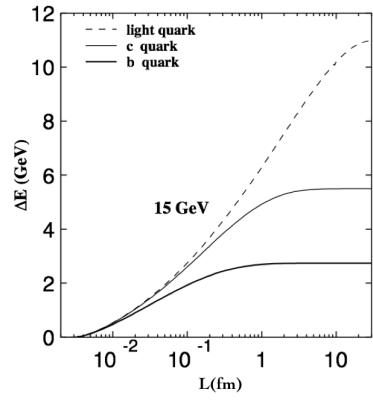

Figure 2. Radiational energy loss of light, $c$ and $b$ quarks having initial energy $E=$ $\sqrt{p_{T}^{2}+m_{q}^{2}}=15 \mathrm{GeV}$, versus path length.

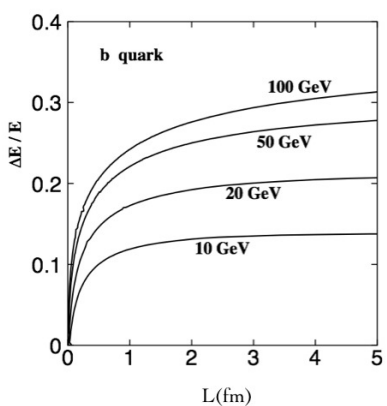

Figure 3. Fractional radiational energy loss by a high- $p_{T} b$ quark, produced with different initial energies.

where $x$ is the fractional light-cone (LC) momentum of the radiated gluon. Apparently, first of all are radiated and regenerated gluons with small longitudinal and large transverse momenta.

The peculiar feature of these jets is closeness of their initial virtuality, imposed by $p_{T}$, and energy $E=\sqrt{p_{T}^{2}+m_{q}^{2}}$. Therefore, increasing the jet energy one unavoidably intensifies radiation and dissipation of energy.

It is instructive to evaluate the amount of energy, radiated after the hard collision by the scattered parton over path length $L$ [2],

$$
\Delta E_{\text {rad }}(L)=E \int_{\Lambda^{2}}^{p_{T}^{2}} d k^{2} \int_{0}^{1} d x x \frac{d n_{g}}{d x d k^{2}} \Theta\left(L-l_{c}\right),
$$

where the radiation spectrum reads,

$$
\frac{d n_{g}}{d x d k^{2}}=\frac{2 \alpha_{s}\left(k^{2}\right)}{3 \pi x} \frac{k^{2}\left[1+(1-x)^{2}\right]}{\left[k^{2}+x^{2} m_{q}^{2}\right]^{2}}
$$

The results of calculations for absolute and fractional radiated energy loss are presented in figures 2 and 3 respectively. We see that radiation of heavy quarks ceases shortly. Only a small fraction of the initial quark energy, $\Delta z=\Delta E_{\text {rad }} / E$, is radiated even after a long time interval. This is quite different from the hadronization pattern for light quarks, which keep radiating long time and lose most of the initial energy (see figure 2). Therefore, the final $B$, or D mesons carry almost the whole momentum of the jet. This expectation is confirmed by the direct measurements of the fragmentation functions in $e^{+} e^{-}$annihilation [3]. The example of the $b \rightarrow B$ fragmentation function depicted in figure 4 indeed shows that the distribution strongly peaks at $z \sim 0.8$. A similar behavior was observed for the $c \rightarrow D$ fragmentation function [4]. At the same time the fragmentation functions of light quarks to light mesons are well known to fall steadily and steeply from small $z$ towards $z=1$ [5]

\section{Production length of heavy mesons}

In what follows we mainly consider for concreteness $B$ meson production. If a $B$-meson (or a $b \bar{q}$ dipole) is produced at the length $l_{p}$, the momentum of $B$ equals to the momentum of the $b$-quark at 


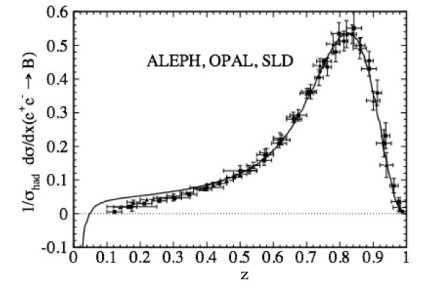

Figure 4. The $b$ fragmentation function, from $e^{+} e^{-}$annihilation. The curve is the DGLAP fit [3].

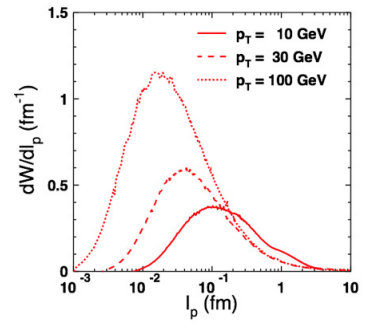

Figure 5. The $l_{p}$-distribution of $B$-mesons produced with different $p_{T}$ in $p p$ collisions.

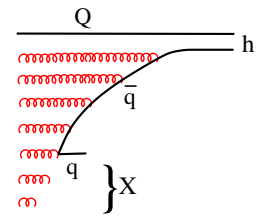

Figure 6. Redistribution of the energy inside a $Q \bar{q}$ dipole by gluon radiation by $Q$ absorbed by $\bar{q}$.

this point. The fraction of the momentum carried by the light $q$ is very small, $x \approx m_{q} / m_{b}$, i.e. is about $5 \%$. We neglect this correction in what follows. Since the the radiational vacuum energy loss $d E / d l$ is known, we can directly relate the production length distribution $W\left(l_{p}\right)$ to the $b \rightarrow B$ fragmentation function $D_{b / B}(z)$,

$$
\frac{d W}{d l_{p}}=\left.\frac{\partial \Delta p_{+}^{b} / p_{+}^{b}}{\partial l}\right|_{l=l_{p}} D_{b / B}(z),
$$

where the production length probability distribution and the fragmentation function are normalized to unity, $\int_{0}^{\infty} d l_{p} d W / d l_{p}=1$ and $\int_{0}^{1} d z D_{b / B}(z)=1$ respectively; $z \equiv p_{+}^{B} / p_{+}^{b}=1-\Delta p_{+}^{b}\left(l_{p}\right) / p_{+}^{b}$; and

$$
\Delta p_{+}^{b}\left(l_{p}\right)=\int_{0}^{l_{p}} d l \frac{d p_{+}^{b}(l)}{d l} .
$$

The rate of LC momentum loss is related to energy loss in accordance with $p_{+}^{b}=E+\sqrt{E^{2}-m_{b}^{2}}$. Thus, knowledge of $D_{b / B}(z)$ and $d E / d l$ gives a direct relation between $z$ and $l_{p}$. As far as we are able to calculate $\Delta z(L)$, we can extract the production length of $B$-mesons directly from data for $D_{b / B}(z)$. The results for $d W / d l_{p}$ are plotted vs $l_{p}$ in figure 5. Remarkably, the mean value of $l_{p}$ shrinks with rising $p_{T}$, like it happens for production of high- $p_{T}$ light hadrons [6].

Concluding, the fragmentation of a $b$-quark in vacuum looks like radiational energy loss up to a point $l=l_{p}$, where it picks up a light $\bar{q}$ forming a colorless $b \bar{q}$ dipole, which performs a direct transition to $B$ without loss the $b$-quark momentum.

\section{In-medium fragmentation}

While in vacuum production of $B$ at $l=l_{p}$ is the end of the story, in a dense medium the $B$-meson (or $b \bar{q}$ dipole) can easily breakup interacting with the medium, and release the $b$-quark, which will continue hadronization and pick-up another light antiquark, and so on. Such recreations and breakups of $B$-mesons will be multiply repeated, until the final production of the detected $B$-meson, which will survive escaping from the medium. We should understand what happens with the $b$-quark, while it propagates either as a constituent of a $b \bar{q}$ dipole, or is released and is losing energy to hadronization.

After the color of the $b$-quark is neutralized by $\bar{q}$, the dipole propagates without radiation and loss of energy. However, if the $b$-quark did not finish regeneration of its color field, it keeps radiating 
inside the dipole. The only difference with the preceding radiation process by a single quark, is that the radiation inside the dipole is reabsorbed by accompanying $\bar{q}$, as is illustrated in figure 6 . Thus, the $b \bar{q}$ dipole does not radiate, its energy remains constant, however the $b$-quark energy is redistributed inside the dipole, decelerating the $b$-quark and accelerating the $\bar{q}$.

As was emphasised above and and demonstrated in figures 2, 3, perturbative radiation of a heavy quark ceases shortly, within a distance of about $1 \mathrm{fm}$ (for a typical $p_{T}$ range). One might think, looking at figure 2 that on longer distances the quark propagates like a free particle with a constant energy. However, this would certainly contradict confinement. A popular model for the non-perturbative mechanism of energy loss is the string model with $d E_{s t r} / d l=-\kappa$, where the string tension in vacuum is $\kappa \approx 1 \mathrm{GeV} / \mathrm{fm}$.

While in vacuum a heavy flavored meson is produced on a very short length scale, $l_{p} \ll 1 \mathrm{fm}$, in a hot medium strong absorption pushes the production point to the dilute medium surface. Therefore non-perturbative energy loss becomes important for such a long-lasting hadronization process, which is continuing throughout the whole area occupied by the medium. However, in a deconfined hot medium no string can be formed. Therefore the magnitude of the string tension, and even its very existence, depends on the medium temperature. We rely on the model $[7,8]$ based on the lattice simulations, of temperature dependence of the string tension, $\kappa(T)=\kappa\left(1-T / T_{c}\right)^{1 / 3}$, where the critical temperature is fixed at $T_{c}=200 \mathrm{MeV}$.

Thus, the full rate of energy loss comes from both perturbative and nonperturbative mechanisms,

$$
\frac{d E}{d l}=\frac{d E_{\text {rad }}}{d l}-\kappa(T) .
$$

After the $b$-quark has promptly radiated the whole spectrum of gluons and decreased its virtuality down to the soft scale, the string becomes the only source of energy loss. If the $b$ quark pick-up a light $\bar{q}$ they are connected by a string, which is also decelerating the $b$-quark and accelerating the $\bar{q}$ with a rate given by the string tension. Such an exchange of energy between $b$ abd $\bar{q}$ is similar to what we observed above for perturbative radiation. So we can conclude that the $b$-quark is constantly loosing energy with a rate, which does not depend of whether it propagates alone, or as a constituent of a $b \bar{q}$ dipole. The process will finalize only after the last recreation of a $B$-meson, which escapes from the medium without further breakups. Apparently, the final $B$-meson will have a reduced energy compared with a $B$-meson produced at $l=l_{p}$ in vacuum, where it has no possibility for further breakups. In other words, with the same starting momentum $p_{+}^{b}$ the final momentum of the $B$ coming out of a medium, will be smaller than in vacuum. This causes suppression because of steeply falling $p_{T}$ distribution of the perturbatively produced $b$-quarks, and due to the steep fall-off of the $b$-quark fragmentation function at small $z$, as is shown in figure 4. Notice that above description of fragmentation and time-dependent energy loss holds for charm quarks as well.

\section{Where does the suppression come from?}

The cross section of production of a $B$-meson with momentum $p_{T}$ in $p p$ collision reads,

$$
\frac{d \sigma(p p \rightarrow B X)}{d^{2} p_{T}}=\int d^{2} p_{+}^{b} \frac{d \sigma(p p \rightarrow Q X)}{d^{2} p_{+}^{b}} \frac{1}{z} D_{b / B}(z),
$$

where $p_{+}^{b}=p_{T}^{b}+\sqrt{\left(p_{T}^{b}\right)^{2}+m_{b}^{2}}$ is the initial LC momentum of the $b$-quark;

$$
z \equiv \frac{\left(p_{T}+\sqrt{p_{T}^{2}+M_{B}^{2}}\right)}{p_{+}^{b}}=1-\frac{\Delta p_{+}^{b}\left(l_{p}\right)}{p_{+}^{b}} .
$$


Similar relation holds for $A A$ collisions, however $l_{p}^{A A}$, the production length of the final, last created colorless $Q \bar{q}$ dipole, is longer than in $p p$ collisions, so LC momentum loss $\Delta p_{+}^{b}$ is larger, and $z_{A A}$ is smaller.

Besides, the cross section acquires a suppression factor $S\left(l_{p}^{A A}\right)$, which is the survival probability of the $Q \bar{q}$ dipole, created at the point $l_{p}^{A A}$, to escape the medium without being broken-up, and to develop the hadronic wave function. Thus in $A A$ collision Eq. (7) is modified as,

$$
\frac{d \sigma(A A \rightarrow B X)}{d^{2} p_{T}}=\int d^{2} p_{+}^{b} \frac{d \sigma(p p \rightarrow Q X)}{d^{2} p_{+}^{b}} \frac{1}{z_{A A}} D_{b / B}\left(z_{A A}\right) S\left(l_{p}^{A A}\right),
$$

with $z_{A A}=1-\Delta p_{+}^{b}\left(l_{p}^{A A}\right) / p_{+}^{b}$.

The last factor $S\left(l_{p}^{A A}\right)$ in (9) is an important player, making the hadronization processes in $p p$ and $A A$ different. Indeed, if this factor were unity, $S=1$, then there would be no reason to delay production point to a longer distance $z_{A A}$ compared with hadronization in vacuum, equation (200). However, absorption terminates the colorless $b \bar{q}$ dipoles produced "too early", so it pushes the production point to the diluted surface of the hot medium, making the production length long, $l_{p}^{A A} \gg l_{p}$, and $z_{A A} \ll z$, resulting in a strong suppression of the fragmentation function $D_{b / B}\left(z_{A A}\right)$ according to figure 4 .

Thus, the two last factors in (9) work in opposite directions, causing suppression of produced $B$ or $D$ mesons:

(i) The fragmentation function $D_{b / B}(z)$, peaking at large $z$ (fig. 4), tends to reduce momentum loss $\Delta p_{+}^{b}\left(l_{p}^{A A}\right)$, selecting shorter $l_{p}^{A A}$.

(ii) However, a shorter $l_{p}^{A A}$ means a longer path length for further propagation of the colorless $Q \bar{q}$ dipole in the medium, increasing its chance to brake-up.

\section{Attenuation of a $Q \bar{q}$ dipole in a hot medium}

A $Q \bar{q}$ pair produced perturbatively with initially small separation, quickly expands. Indeed, the light quark in the $Q \bar{q}$-meson carries a tiny fraction of the momentum, $x \sim m_{q} / m_{Q}$. Therefore, even if the produced $b \bar{q}$ dipole has a small transverse separation, its size expands with a high speed, enhanced by $1 / x$. The formation time of the $B$-meson wave function (in the medium rest frame) is very short,

$$
t_{f}^{B}=\frac{\sqrt{p_{T}^{2}+m_{B}^{2}}}{2 m_{B} \omega},
$$

where $\omega=300 \mathrm{MeV}$ is the oscillator frequency, which determines the splitting of the ground state and the first radial excitation. For instance at $p_{T}=10 \mathrm{GeV}$ the $B$ meson is formed on a distance $l_{f}^{B}=0.8 \mathrm{fm}$.

Thus, not a small-size $r^{2} \sim 1 / p_{T}^{2}$ dipole, like for light hadron production [9], but a nearly formed large $Q \bar{q}$ dipole is propagating through the medium. It can be easily broken-up, so its mean free path is quite short. Indeed, the $B$-meson is nearly as big as a pion, $\left\langle r_{c h}^{2}\right\rangle_{B}=0.378 \mathrm{fm}^{2}$ [10]. The mean free path of such a meson in a hot medium is very short, $\lambda_{B} \sim\left[\hat{q}\left\langle r_{T}^{2}\right\rangle\right]^{-1}$, where $\left\langle r_{T}^{2}\right\rangle=8\left\langle r_{c h}^{2}\right\rangle / 3$. The so called transport coefficient $\hat{q}$ is the rate of broadening of the quark transverse momentum in the medium, this is why it controls the dipole absorption cross section. For instance, at $\hat{q}=1 \mathrm{GeV}^{2} / \mathrm{fm}$ (compare with [9]) the mean free pass $\lambda_{B}=0.04 \mathrm{fm}$, i.e. the $b$-quark propagates through the hot medium, frequently picking up and losing light antiquark comovers. Meanwhile the $b$-quark keeps losing energy with a rate, enhanced by medium-induced effects. Eventually the detected $B$-meson is formed and can survive in the dilute periphery of the medium. 


\subsection{The suppression factor $S\left(l_{p}^{A A}\right)$}

Thus, the finally detected $B$-meson is produced at $l=l_{p}^{A A}$. In the low energy limit the formation length (10) is very short and one can use the eikonal Glauber approximation,

$$
S\left(l_{p}^{A A}\right)=\exp \left[-\frac{\left\langle r_{B}^{2}\right\rangle}{2} \int_{l_{p}^{A A}}^{\infty} d l \hat{q}(l)\right]
$$

In contrast, in another extreme, the high-energy limit, the dipole size is "frozen" by Lorentz time dilation. Then,

$$
S\left(l_{p}^{A A}\right)=\int d^{2} r d x\left|\Psi_{B}(r, x)\right|^{2} \exp \left[-\frac{r^{2}}{2} \int_{l_{p}^{A A}}^{\infty} d l \hat{q}(l)\right],
$$

where $\Psi_{B}(r, x)$ is the $\mathrm{LC}$ wave function of the $B$-meson.

The general description interpolating between these two limits, is the path-integral technique [11], summing all paths of the $Q$ and $\bar{q}$. The result has the form,

$$
S\left(l_{1}, l_{2}\right) \propto\left|\int_{0}^{1} d x \int d^{2} r_{1} d^{2} r_{2} \Psi_{M}^{\dagger}\left(r_{2}, x\right) G_{Q \bar{q}}\left(l_{1}, \vec{r}_{1}, x ; l_{2}, \vec{r}_{2}, x\right) \Psi_{i n}\left(r_{1}, x\right)\right|^{2},
$$

where in the case under consideration $l_{1}=l_{p}^{A A}, l_{2} \rightarrow \infty$. The initial distribution amplitude $\Psi_{i n}\left(r_{1}, x\right)$ is taken in the Gaussian form with mean separation $\left\langle r_{1}^{2}\right\rangle=\left\langle r_{B}^{2}\right\rangle$. The Green function $G_{Q \bar{q}}\left(l_{1}, \vec{r}_{1}, x ; l_{2}, \vec{r}_{2}, x\right)$ describing propagation of the dipole between longitudinal coordinates $l_{1}, l_{2}$ with initial and final separations $\vec{r}_{1}$ and $\vec{r}_{2}$ respectively, satisfies the 2-dimensional LC equation,

$$
i \frac{d}{d l_{2}} G_{Q \bar{q}}\left(l_{1}, \vec{r}_{1}, x ; l_{2}, \vec{r}_{2}, x\right)=\left[-\frac{\Delta_{r_{2}}}{2 p_{T} x(1-x)}+V_{Q \bar{q}}\left(l_{2}, \vec{r}_{2}\right)\right] G_{Q \bar{q}}\left(l_{1}, \vec{r}_{1} ; l_{2}, \vec{r}_{2}\right)
$$

The imaginary part of the LC potential is responsible for absorption, $\operatorname{Im} V_{Q \bar{q}}(l, \vec{r})=-\hat{q}(l) r^{2} / 4$. The real part is the phenomenological Cornell-type potential, adjusted to reproduce the masses and decay constants for $B$ and $D$ mesons $[10,12]$.

\section{Results}

While the employed phenomenology allows a parameter-free description of data, one parameters is unavoidably present in such kind of analysis. This is the transport coefficient $\hat{q}$, which cannot be reliably predicted, in particular its coordinate and time dependence. We employ here the popular model [13],

$$
\hat{q}(l, \vec{b}, \vec{\tau})=\frac{\hat{q}_{0} l_{0}}{l} \frac{n_{\text {part }}(\vec{b}, \vec{\tau})}{n_{\text {part }}(0,0)} \Theta\left(l-l_{0}\right)
$$

where $\vec{b}$ is the impact parameter of nuclear collision, $\vec{\tau}$ is the impact parameter of the hard partonparton collision relative to the center of one of the nuclei, $n_{\text {part }}(\vec{b}, \vec{\tau})$ is the number of participants, and $\hat{q}_{0}$ is the rate of broadening of a quark propagating in the maximal medium density produced at impact parameter $\tau=0$ in central collisions $(b=0)$ at the time $t=t_{0}$ after the collision. The time interval 
after the hard collision is $t=l / v_{Q \bar{q}}$ where $v_{Q \bar{q}}$ is the speed of the $Q \bar{q}$ dipole. We fixed the medium equilibration time at $t_{0}=1 \mathrm{fm}$.

In such a scheme $\hat{q}_{0}$ is the only fitted parameter, which, however, has been already determined from other hard processes in $A A$ collisions. In particular, it the analysis [9] of data on quenching of light high- $p_{T}$ hadrons it was found $\hat{q}_{0}=1.6 \mathrm{GeV}^{2} / \mathrm{fm}$ at $\sqrt{s}=200 \mathrm{GeV}$ and $\hat{q}_{0}=2 \mathrm{GeV}^{2} / \mathrm{fm}$ at $\sqrt{s}=2760 \mathrm{GeV}$.

Different sources of time-dependent medium-induced energy loss were added, including radiative and collisional mechanisms [14]. Medium-induced energy loss is much smaller than the vacuum one, and do not produce a dramatic effect. They are particularly small for heavy flavors.

The results of calculations for $B$-meson production are compared with data on indirect production of $J / \psi$, originating from $B$ decays. Comparison is made vs $p_{T}$ and centrality. In figure 7 the dashed curve is calculated with pure vacuum energy loss (radiative plus string) neglecting induced energy loss for $\hat{q}_{0}=2.5$. The upper and bottom solid curves are calculated with $\hat{q}_{0}=2.5$ and $3 \mathrm{GeV}^{2} / \mathrm{fm}$ respectively, at $\sqrt{s}=2.76 \mathrm{TeV}$ in comparison with data [15]. Figure 8 demonstrate comparison
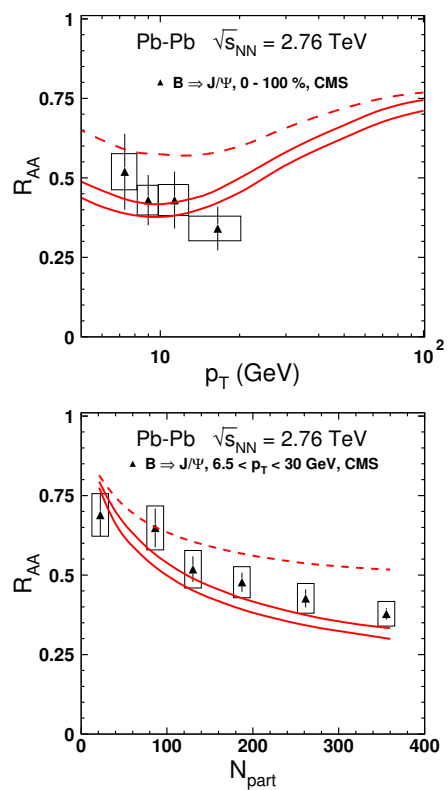

Figure 7. Comparison with CMS data for indirect $J / \psi$ production [16] at $\sqrt{s}=2.76 \mathrm{TeV}$. The dashed curves are calculated neglecting induced energy loss. The upper and bottom solid curves are calculated with $\hat{q}_{0}=2.5$ and $3 \mathrm{GeV}^{2} / \mathrm{fm}$ respectively.
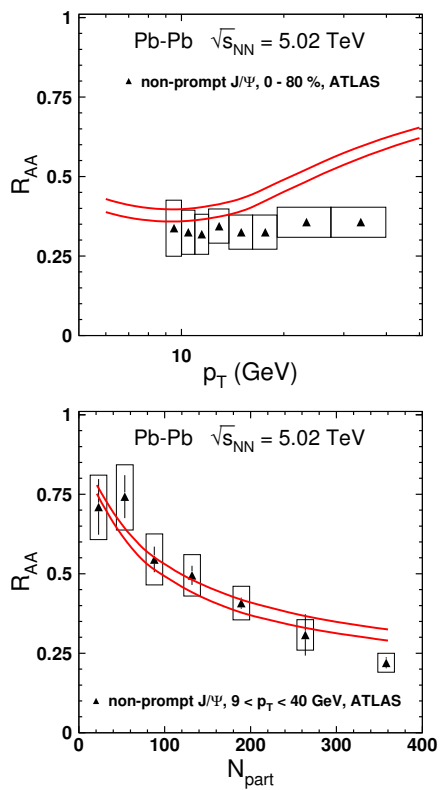

Figure 8. The same as in Fig. 7, but at $\sqrt{s}=$ $5.02 \mathrm{TeV}$. The upper and bottom curves are calculated with $\hat{q}_{0}=2.6$ and $3.2 \mathrm{GeV}^{2} /$ fm respectively. Data are from [16].

with recent data from ATLAS [16]. The upper and bottom curves are calculated with $\hat{q}_{0}=2.6$ and $3.2 \mathrm{GeV}^{2} / \mathrm{fm}$ respectively, at $\sqrt{s}=5.02 \mathrm{TeV}$. The lack of rise of $R_{A A}$ at high $p_{T}$ in data looks unusual, compared with the typical behavior of other hadrons, so we would restrain of claiming a serious disagreement. 
Notice that the rise of $\hat{q}_{0}$ with energy is natural, but its value, should not depend on the process used to measure it. However, different analyses of different reactions rely on many simplifications and model-dependent assumptions. Therefore, would be naive to expect exact correspondence of $\hat{q}_{0}$ values extracted from data on different hard processes. The values found here are pretty close to those extracted in [9] from data on high- $p_{T}$ production of light hadrons.

The approach developed here can also be applied to production of $D$-mesons. The results are compared with data in figures 9 and $10 \mathrm{vs} p_{T}$ and centrality.
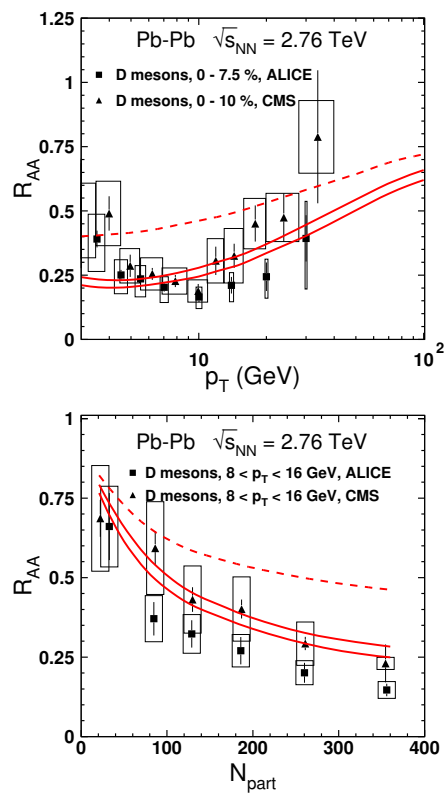

Figure 9. The same as in figure 7 , but for $D$-mesons at $\sqrt{s}=2.76 \mathrm{TeV}$. Calculations are done with the same values of $\hat{q}_{0}=2.5$ and $3 \mathrm{GeV}^{2} / \mathrm{fm}$. Data are from [17-19] and $[20,21]$.
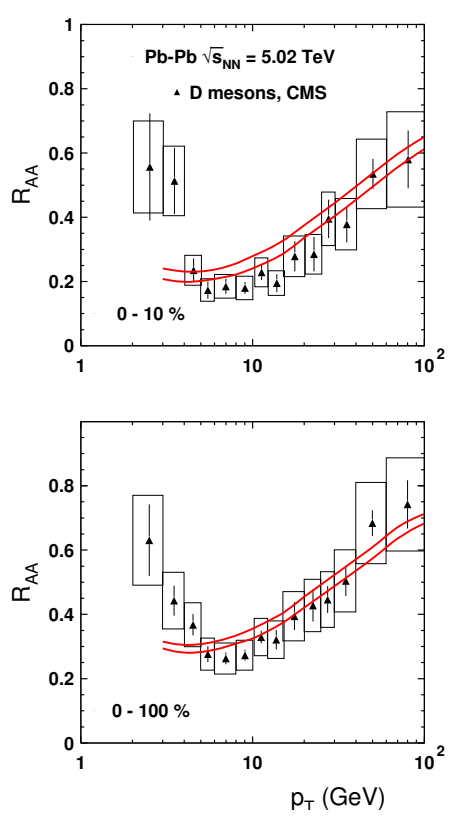

Figure 10. The same as in figure 9 , but at $\sqrt{s}=5.02 \mathrm{TeV}$ for centralities $0-80 \%$ and minimum bias events. Data from CMS measurements [22].

Notice that $c$-quarks radiate in vacuum much more energy than $b$-quarks, while the effects of absorption of $c \bar{q}$ and $b \bar{q}$ dipoles in the medium are similar. Therefore, $D$-mesons are suppressed in $A A$ collisions more than $B$-mesons.

\section{Summary}

Fragmentation of high- $p_{T}$ heavy quarks expose nontrivial features.

- Heavy and light quarks produced in high- $p_{T}$ partonic collisions radiate differently. Heavy quarks regenerate their stripped-off color field much faster than light ones, and radiate a significantly smaller fraction of the initial energy.

- This peculiar feature of heavy-quark jets leads to a specific shape of the fragmentation functions. Differently from light flavors, the heavy quark fragmentation functions strongly peak at large frac- 
tional momentum $z$, i.e. the produced heavy-light meson, $B$ or $D$, carry the main fraction of the jet momentum. This is a clear evidence of a short production time of heavy-light mesons.

- Contrary to the propagation of a small $\bar{q} q$ dipole, which survives in the medium due to color transparency, a $\bar{q} Q$ dipole promptly expands to a large size. Such a big dipole has no chance to survive intact in a hot medium. On the other hand, a breakup of such a dipole does not suppress directly the production rate of $\bar{q} Q$ colorless dipoles, but increase energy loss preceding the final production of a heavy flavored meson. This is different from the scenario of high- $p_{T}$ production of light $\bar{q} q$ mesons [9].

- Data for production of high- $p_{T} B$ and $D$ mesons are explained in a parameter-free way. The extracted values of the transport coefficient agree with the results of previous analyses within unavoidable uncertainties, related to employed simplifications and model dependent assumptions, made in the calculations.

- We have disregarded so far the small initial state suppression of heavy flavors due to higher twist heavy dipole attenuation and leading twist shadowing [23]. Inclusion of these effect will lead to a small decrease of the values of $\hat{q}_{0}$ extracted from the analysis.

\section{Acknowledgements}

This work was supported in part by Fondecyt (Chile) grants 1130543, 1130549, 1140842, 1140377, by Proyecto Basal FB 0821 (Chile), and by CONICYT grant PIA ACT1406 (Chile). J.N. work was partially supported by the grant 13-20841S of the Czech Science Foundation (GAČR), by the Grant MŠMT LG15001, by the Slovak Research and Development Agency APVV-0050-11 and by the Slovak Funding Agency, Grant 2/0020/14.

\section{References}

[1] Y. L. Dokshitzer and D. E. Kharzeev, Phys. Lett. B 519, 199 (2001); [hep-ph/0106202].

[2] B. Z. Kopeliovich, I. K. Potashnikova and I. Schmidt, Phys. Rev. C 82, 037901 (2010).

[3] B. A. Kniehl, G. Kramer, I. Schienbein and H. Spiesberger, Phys. Rev. D 77, 014011 (2008); [arXiv:0705.4392 [hep-ph]].

[4] T. Kneesch, B. A. Kniehl, G. Kramer and I. Schienbein, Nucl. Phys. B 799, 34 (2008); [arXiv:0712.0481 [hep-ph]].

[5] B. A. Kniehl, G. Kramer and B. Potter, Nucl. Phys. B 597, 337 (2001); [hep-ph/0011155].

[6] B. Z. Kopeliovich, H. -J. Pirner, I. K. Potashnikova and I. Schmidt, Phys. Lett. B 662, 117 (2008).

[7] H. Toki, S. Sasaki, H. Ichie and H. Suganuma, Austral. J. Phys. 50, 199 (1997); [hep-ph/9602367].

[8] H. Ichie, H. Suganuma and H. Toki, Phys. Rev. D 54, 3382 (1996); [hep-ph/9602412].

[9] B. Z. Kopeliovich, J. Nemchik, I. K. Potashnikova and I. Schmidt, Phys. Rev. C 86, 054904 (2012); [arXiv:1208.4951 [hep-ph]].

[10] C. W. Hwang, Eur. Phys. J. C 23, 585 (2002); [hep-ph/0112237].

[11] B. Z. Kopeliovich and B. G. Zakharov, Phys. Rev. D 44, 3466 (1991).

[12] M. Z. Yang, Eur. Phys. J. C 72, 1880 (2012); [arXiv:1104.3819 [hep-ph]].

[13] X. F. Chen, C. Greiner, E. Wang, X. N. Wang and Z. Xu, Phys. Rev. C 81, 064908 (2010); [arXiv:1002.1165 [nucl-th]].

[14] R. Baier, Nucl. Phys. A 715, 209 (2003); [hep-ph/0209038].

[15] S. Chatrchyan et al. (CMS Collaboration); JHEP 1205, 063 (2012); arXiv:1201.5069 [nucl-ex]. CMS-PAS-HIN-12-014. 
[16] ATLAS-CONF-2016-109 (2016).

[17] A. Dubla (for the ALICE Collaboration) "Measurements of heavy-flavour nuclear modification factor and elliptic flow in $P b-P b$ collisions at $\sqrt{s_{N N}}=2.76 \mathrm{TeV}$ with ALICE", Quark Matter 2015, Sep. 27 - Oct. 3, 2015, Kobe, Japan.

[18] J. Adam et al. (ALICE Collaboration); JHEP 03, 081 (2016); arXiv:1509.06888 [nucl-ex], CERN-PH-EP-2015-25.

[19] J. Adam et al. (ALICE Collaboration); JHEP 1511, 205 (2015) arXiv:1506.06604 [nucl-ex], CERN-PH-EP-2015-151.

[20] Jian Sun (for the CMS Collaboration); "Open Heavy Flavor Measurements in Heavy Ion Collisions with CMS", Quark Matter 2015, Sep. 27 - Oct. 3, 2015, Kobe, Japan.

[21] S. Chatrchyan at al. (CMS Collaboration); "Nuclear Modification Factor of prompt $D_{0}$ in $\mathrm{Pb}-\mathrm{Pb}$ Collisions at $\sqrt{s_{N N}}=2.76$ TeV" CMS-PAS-HIN-15-005.

[22] A.M. Sirunyan et al. (the CMS Collaboration) $D^{0}$ meson nuclear modification factor in $\mathrm{Pb}-\mathrm{Pb}$ collisions at $\sqrt{s_{N N}}=5.02 \mathrm{TeV} "$, CMS PAS HIN-16-001.

[23] B. Z. Kopeliovich and A. V. Tarasov, Nucl. Phys. A 710, 180 (2002) [hep-ph/0205151]. 